\title{
Shape Preserving Interpolation Using Rational Cubic Ball Function and Its Application in Image Interpolation
}

\author{
Samsul Ariffin Abdul Karim ${ }^{1}$ and Azizan Saaban ${ }^{2}$ \\ ${ }^{1}$ Fundamental and Applied Sciences Department, Universiti Teknologi PETRONAS, Bandar Seri Iskandar, 32610 Seri Iskandar, \\ Perak Darul Ridzuan, Malaysia \\ ${ }^{2}$ College of Arts and Sciences, Universiti Utara Malaysia, 06010 Sintok, Kedah, Malaysia
}

Correspondence should be addressed to Samsul Ariffin Abdul Karim; samsul_ariffin@utp.edu.my

Received 3 July 2017; Revised 18 September 2017; Accepted 4 October 2017; Published 13 November 2017

Academic Editor: Suzanne M. Shontz

Copyright ( 2017 Samsul Ariffin Abdul Karim and Azizan Saaban. This is an open access article distributed under the Creative Commons Attribution License, which permits unrestricted use, distribution, and reproduction in any medium, provided the original work is properly cited.

\begin{abstract}
New rational cubic Ball interpolation with one parameter is proposed for shape preserving interpolation such as positivity, monotonicity, and convexity preservations and constrained data lie on the same side of the given straight line. To produce shape preserving interpolant, the data dependent sufficient condition is derived on the parameter. The rational bicubic Ball function is constructed by using tensor product approach and it will be used for application in image upscaling. Numerical and graphical results are presented by using Mathematica and MATLAB including comparison with some existing scheme.
\end{abstract}

\section{Introduction}

Shape preserving interpolation and approximation is important in many sciences and engineering applications. Common strategy in shape preserving interpolation is the construction of rational or nonrational interpolant that satisfies some shape preserving criteria. When the interpolant satisfies the conditions, then the resulting interpolating curve or approximating curve preserves the shape of the given data. For instance, if the given data is positive, then the interpolating curve is positive on the whole interval. There is many good research in this area. For instance, Abbas et al. [1] discussed the application of rational cubic spline for image interpolation based on genetic algorithm (GA). Abbas et al. [2] use similar idea as in [1] but they replace the rational cubic spline with rational cubic Ball interpolation.

Majeed et al. [3] discussed the craniofacial reconstruction by using rational cubic Ball with cubic denominator without any free parameters. They utilized the original Ball function from Ball [4]. The main difference between their scheme and the proposed scheme in this study is that we construct rational cubic Ball spline with quadratic denominator with one parameter. Thus the computation time will be less compared with the work of Majeed et al. [3] (Table 7). Besides that, there are different types of rational cubic Ball interpolant. For instance, Karim [5, 6] uses different form from what we proposed in this study. The main contributions of this study can be summarized as follos:

(i) The proposed scheme does not require the modification of the first derivative if shape preserving is violated as shown in the work of Brodlie and Butt [7].

(ii) The proposed scheme is guaranteed to produce positive and convex curves for positive and convex data sets while the scheme by Hussain et al. [8,9] might be producing nonpositivity interpolating curve on some interval.

(iii) The proposed scheme requires less computation time compared to the bicubic spline interpolation for image upscaling.

This paper is organized as follows. After the Introduction to the subject matter, Methodology will be discussed in Section 2. This includes the construction of the new rational cubic Ball interpolation and the derivation of the sufficient condition of the rational cubic Ball for constrained data interpolation, monotonicity, and convexity preservation, respectively. Numerical and graphical results are given in 
Section 3. Section 4 is devoted for application of rational bicubic Ball function for image interpolation. Conclusions are given in the final section.

\section{Methodology}

This section discusses the construction of new rational cubic Ball (cubic/quadratic) with one parameter.

2.1. Rational Cubic Ball Spline Interpolant. Given the data $\left\{\left(x_{i}, f_{i}\right), i=0,1, \ldots, n\right\}$ and first derivative $d_{i}, i=0,1, \ldots, n$ with $x_{0}<x_{1}<\cdots<x_{n}$, let $h_{i}=x_{i+1}-x_{i}, \Delta_{i}=\left(f_{i+1}-f_{i}\right) / h_{i}$ and $\theta=\left(x-x_{i}\right) / h_{i}$; thus $0 \leq \theta \leq 1$. On each subinterval $x \in\left[x_{i}, x_{i+1}\right], i=0,1, \ldots, n-1$, the new rational cubic Ball interpolant with shape parameter $\alpha_{i} \geq 0$ is defined by

$$
S_{i}(x)=\frac{P_{i}(\theta)}{Q_{i}(\theta)},
$$

where

$$
\begin{aligned}
P_{i}(\theta)= & (1-\theta)^{2} f_{i}+(1-\theta)^{2} \theta A_{i}+(1-\theta) \theta^{2} B_{i} \\
& +\theta^{2} f_{i+1}, \\
Q_{i}(\theta)= & 1+\alpha_{i}(1-\theta) \theta .
\end{aligned}
$$

The rational cubic Ball interpolant in (1) is $C^{1}$ continuity at the knots $x_{i}, i=1,2, \ldots, n-1$. Mathematically this can be rewritten as

$$
\begin{gathered}
S\left(x_{i}\right)=f_{i}, \quad S^{(1)}\left(x_{i}\right)=d_{i} \\
S\left(x_{i+1}\right)=f_{i+1}, \quad S^{(1)}\left(x_{i+1}\right)=d_{i+1},
\end{gathered}
$$

where $S^{(1)}\left(x_{i}\right)$ denotes the first-order derivative with respect to $x$ at the knot $x_{i}$. From conditions (3), by simple derivation using (1), the unknown variables, $A_{i}, B_{i} i=0,1, \ldots, n-1$, are given as

$$
\begin{aligned}
A_{i} & =\left(\alpha_{i}+2\right) f_{i}+h_{i} d_{i}, \\
B_{i} & =\left(\alpha_{i}+2\right) f_{i+1}-h_{i} d_{i+1} .
\end{aligned}
$$

It can be verified that, at $\theta=0, S^{(1)}\left(x_{i}\right)=d_{i}$ and similarly at $\theta=1, S^{(1)}\left(x_{i+1}\right)=d_{i+1}$.

2.2. Derivative Estimation. There are many methods to estimate the first derivative $d_{i}, i=0,1, \ldots, n$, for instance, the geometric mean method (GMM), harmonic mean method (HMM), and arithmetic mean method (AMM). The common method is arithmetic mean method (AMM) (Karim $[5,6])$. In this study we adopted AMM since it is simple to use and suitable for all types of data set. The derivation is given as follows $[5,6]$.

2.2.1. Arithmetic Mean Method. The values of $d_{i}$ are given as

$$
d_{i}=\frac{h_{i-1} \Delta_{i}+h_{i} \Delta_{i-1}}{h_{i-1}+h_{i}}, \quad i=1,2,3, \ldots, n-1 .
$$

TABLE 1: Data set for shape control analysis.

\begin{tabular}{cccccc}
\hline$i$ & 0 & 1 & 2 & 3 & 4 \\
\hline$x_{i}$ & 0 & 2 & 3 & 9 & 11 \\
$f_{i}$ & 0.5 & 1.5 & 7 & 9 & 13 \\
$d_{i}$ & -2.833 & 3.833 & 4.762 & 1.583 & 2.417 \\
\hline
\end{tabular}

The derivative value of $d_{0}$ and $d_{n}$ is given as

$$
\begin{aligned}
& d_{0}=\Delta_{0}+\left(\Delta_{0}-\Delta_{1}\right)\left(\frac{h_{0}}{h_{0}+h_{1}}\right), \\
& d_{n}=\Delta_{n-1}+\left(\Delta_{n-1}-\Delta_{n-2}\right)\left(\frac{h_{n-1}}{h_{n-1}+h_{n-2}}\right) .
\end{aligned}
$$

The first derivative value $d_{i}, i=0,1, \ldots, n$ is estimates from (5), (6), and (6). For data point $\left\{\left(x_{i}, f_{i}\right), i=0,1, \ldots, n\right\}$, the first derivative value, $d_{i}, i=0,1, \ldots, n$, and shape parameter $\alpha_{i} \geq 0$, the rational cubic Ball, $S_{i}(x)$, defined by (1) can be constructed.

2.2.2. Shape Control Analysis. Obviously when $\alpha_{i}=0$, the proposed rational cubic Ball interpolant is reduced to cubic Ball polynomial as

$$
\begin{aligned}
S_{i}(x)= & f_{i}(1-\theta)^{2}+\left(2 f_{i}+h_{i} d_{i}\right)(1-\theta)^{2} \theta \\
& +\left(2 f_{i+1}-h_{i} d_{i+1}\right)(1-\theta)^{2} \theta+f_{i+1} \theta^{2} .
\end{aligned}
$$

The piecewise rational cubic Ball $S_{i}(x)$ defined by (1) can also be rewritten as

$$
\begin{aligned}
S_{i}(x) & \\
\qquad & (1-\theta) f_{i}+\theta f_{i+1} \\
& +\frac{\left[\Delta_{i}(2 \theta-1)+(1-\theta) d_{i}-\theta d_{i+1}\right] h_{i}(1-\theta) \theta}{Q_{i}(\theta)} .
\end{aligned}
$$

When $\alpha_{i} \rightarrow \infty$, the rational cubic interpolant $S_{i}(x)$ reduces to a straight line on the subinterval $\left[x_{i}, x_{i+1}\right], i=0,1, \ldots, n-1$

$$
\lim _{\alpha_{i} \rightarrow \infty} S_{i}(x)=(1-\theta) f_{i}+\theta f_{i+1} .
$$

To avoid the denominator in (1) becoming zero, we restrict the parameter as $\alpha_{i} \geq 0$ for $i=0,1, \ldots, n-1$.

Shape control analysis can further be elucidated by using a graphical approach. To see the effect of the shape control, by varying the value of shape parameter $\alpha_{i} \geq 0, i=0,1, \ldots, n-1$, the data given in Table 1 is used.

2.3. Numerical Example 1: Shape Control Analysis. Figure 1 shows the shape control analysis. For Figure 1(a) the value for shape parameter $\alpha_{i}, i=0,1,2,3,4$ are 0.1 (black), 10 (dashed), and 100 (gray). The higher the value of the parameter $\alpha_{i}$ is, $i=0,1,2,3,4$, the more loose the interpolating curve tends to be. Meanwhile for Figure 1(b) the values for shape parameter are $\alpha_{i}=0$ (black), cubic polynomial Ball, that is, from (8), and $\alpha_{i}=1000$ (dashed). Equation (10) is verified by Figure 1(b), that is, the rational interpolant converging to a straight line when $\alpha_{i}, i=0,1,2,3,4$ is a larger positive number. 


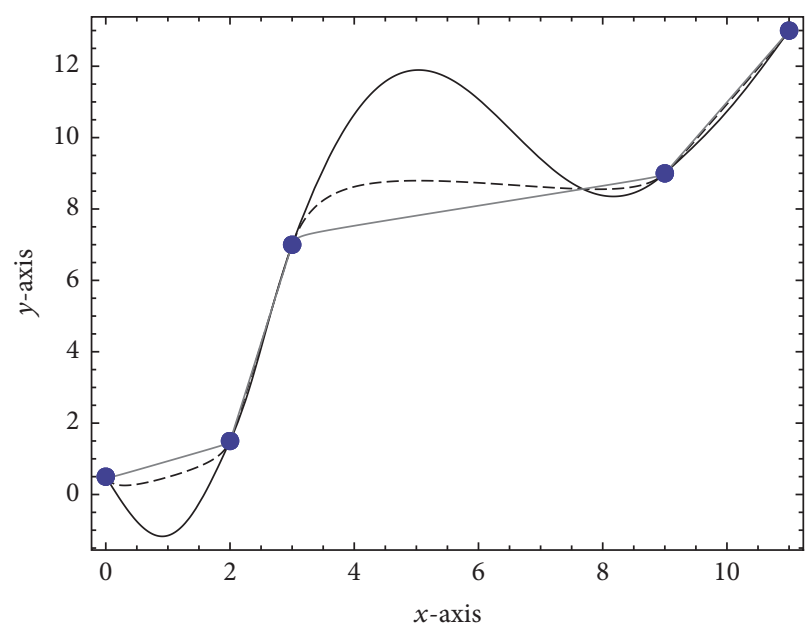

(a) With $\alpha_{i}=0.1$ (black), $\alpha_{i}=10$ (dashed), and $\alpha_{i}=100$ (gray)

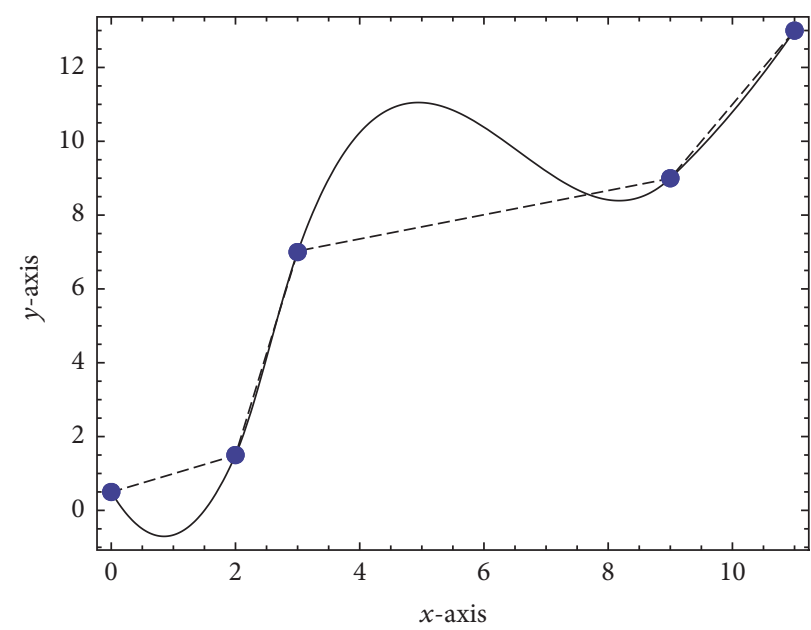

(b) With $\alpha_{i}=0$ (black) and $\alpha_{i}=1000$ (dashed)

FIgURE 1: Shape control analysis.

2.4. Application in Shape Preserving Interpolation. In this Section, we discuss the application of the proposed rational cubic Ball function for shape preserving interpolation. The data under consideration are positive, monotone, convex, and on the same side as the constraint line.

2.5. Positivity Preserving. In this study, the given data is strictly positive to avoid dividing by zero such that

$$
f_{i}>0, \quad i=1,2, \ldots, n .
$$

The main objective is to find the value of shape parameter $\alpha_{i} \geq 0, i=0,1, \ldots, n-1$ that will ensure that rational interpolant defined by (1) is positive to produce the positive interpolating curve on the whole given domain. Since, for all $\alpha_{i} \geq 0, i=0,1, \ldots, n-1$, the denominator $Q_{i}(\theta)>0$, therefore, the positivity of the rational cubic interpolant in (1) depends on the cubic polynomial $P_{i}(\theta), i=0,1, \ldots, n-1$; that is, $P_{i}(\theta)>0$. Schmidt and Hess [11] have developed the result for the positivity of $P_{i}(\theta), i=0,1, \ldots, n-1$ as

$$
\left(P_{i}^{\prime}(0), P_{i}^{\prime}(1)\right) \in R_{1} \cup R_{2},
$$

where

$$
\begin{aligned}
R_{1} & =\left\{(a, b): a>\frac{-3 P_{i}(0)}{h_{i}}, b<\frac{3 P_{i}(1)}{h_{i}}\right\}, \\
R_{2} & =\{(a, b): \\
& 36 f_{i} f_{i+1}\left(a^{2}+b^{2}+a b-3 \Delta_{i}(a+b)+3 \Delta_{i}^{2}\right) \\
& +3\left(f_{i+1} a-f_{i} b\right)\left(2 h_{i} a b-3 f_{i+1} a+3 f_{i} b\right) \\
& \left.+4 h_{i}\left(f_{i+1} a^{3}-f_{i} b^{3}\right)-h_{i}^{2} a^{2} b^{2}>0\right\} .
\end{aligned}
$$

Notation $P_{i}^{\prime}(0), P_{i}^{\prime}(1)$ indicates the first derivative of $P$ with respect to $x$ at $x_{i}$ and $x_{i+1}$ (i.e., at $\theta=0$ and $\theta=1$, resp.). Note that $a=P_{i}^{\prime}(0)$ and $b=P_{i}^{\prime}(1)$, respectively [11].
The following theorem gives the sufficient conditions for the positivity of the rational cubic Ball interpolant. It is data dependent and has one free parameter to alter the final positive interpolating curves.

Theorem 1. For a strictly positive data defined in (11), the rational cubic Ball interpolant on $\left[x_{0}, x_{n}\right]$ is positive if, in each subinterval $\left[x_{i}, x_{i+1}\right], i=0,1, \ldots, n-1$, if the following sufficient conditions are satisfied:

$$
\alpha_{i}>\max \left\{0,-\frac{h_{i} d_{i}}{f_{i}}, \frac{h_{i} d_{i+1}}{f_{i+1}}\right\} .
$$

Proof. The sufficient condition of the positivity is derived from condition (13) as follows:

$$
\begin{aligned}
P_{i}^{\prime}(0) & >\frac{-3 P_{i}(0)}{h_{i}} \Longrightarrow \\
\frac{-2 f_{i}+V_{i}}{h_{i}} & >\frac{-3 f_{i}}{h_{i}}, \\
P_{i}^{\prime}(1) & <\frac{3 P_{i}(1)}{h_{i}} \Longrightarrow \\
\frac{2 f_{i+1}-W_{i}}{h_{i}} & <\frac{3 f_{i+1}}{h_{i}} .
\end{aligned}
$$

Both conditions can be further simplified as follows:

$$
\begin{gathered}
\alpha_{i}>-\frac{h_{i} d_{i}}{f_{i}}, \\
\alpha_{i}>\frac{h_{i} d_{i+1}}{f_{i+1}} .
\end{gathered}
$$

The sufficient condition the positivity of the rational cubic Ball defined by (1) can be obtained by combining conditions (17) and (18) into one condition given as follows:

$$
\alpha_{i}>\max \left\{0,-\frac{h_{i} d_{i}}{f_{i}}, \frac{h_{i} d_{i+1}}{f_{i+1}}\right\} .
$$


For the purpose of computer implementation, the above condition can be rewritten as follows:

$$
\alpha_{i}=\lambda_{i}+\max \left\{0,-\frac{h_{i} d_{i}}{f_{i}}, \frac{h_{i} d_{i+1}}{f_{i+1}}\right\}
$$

such that $\lambda_{i}>0$ satisfies $0<\lambda_{i} \leq 0.25$.

Algorithm for computer implementation is as follows.

\section{Algorithm 2.}

Input. Data points, first derivative, and parameter $\lambda_{i}>0$.

Output. Positive interpolating curve.

Step 1. Input data points $\left\{\left(x_{i}, f_{i}\right), i=0,1,2, \ldots, n\right\}$.

Step 2. For $i=1, \ldots, n$

(i) calculate the first derivative values by using (8), (9), and (10).

Step 3. For $i=1, \ldots, n-1$

(i) calculate the parameter values, $\alpha_{i}>0$ by using the sufficient condition given in (20);

(ii) repeat by choosing different value of $\lambda_{i}>0$; that is, $0<\lambda_{i} \leq 0.25$.

Step 4. For $i=1, \ldots, n-1$

(i) construct the positive interpolating curve with $C^{1}$ continuity.

2.6. Constrained Data Interpolation. This section discusses the construction of the sufficient condition for constrained data interpolation for data that lies on the same side as the given constraint line. There are three types for constraint line such as above straight line, below straight line, and in between two lines. In this paper, we will only study the constrained interpolation for the data lie above arbitrary constraint line. The problem statement can be read as follows.

Given data set $\left\{\left(x_{i}, f_{i}\right), i=0,1, \ldots, n\right\}$ which lies above the straight line, that is, $y=m x+c$, such that

$$
f_{i}>m x_{i}+c, \quad i=0,1, \ldots, n,
$$

by rewriting the arbitrary straight line in parametric form, then, (21) is equal to

$$
S_{i}(x)>k_{i}(1-\theta)+l_{i} \theta
$$

with $k_{i}=m x_{i}+c, i=0,1, \ldots, n$ and $l_{i}=m x_{i+1}+c, i=$ $0,1, \ldots, n-1$, respectively.

Equation (22) can be further simplified to

$$
S_{i}(x)=\frac{P_{i}(\theta)}{Q_{i}(\theta)}>k_{i}(1-\theta)+l_{i} \theta, \quad i=0,1, \ldots, n-1
$$

or equivalently

$$
S_{i}(x)=\frac{U_{i}(\theta)}{Q_{i}(\theta)}>0
$$

where

$$
U_{i}(\theta)=P_{i}(\theta)-\left(k_{i}(1-\theta)+l_{i} \theta\right) Q_{i}(\theta) .
$$

By simple derivation we obtain

$$
\begin{aligned}
U_{i}(\theta)= & (1-\theta)^{2}\left(f_{i}-k_{i}\right)+(1-\theta)^{2} \theta C_{i 1} \\
& +(1-\theta) \theta^{2} C_{i 2}+\theta^{2}\left(f_{i+1}-l_{i}\right)
\end{aligned}
$$

with

$$
\begin{aligned}
& C_{i 1}=f_{i}+\alpha_{i}\left(f_{i}-k_{i}\right)+\left(f_{i}-k_{i}\right)+h_{i} d_{i}-l_{i}, \\
& C_{i 2}=f_{i+1}+\alpha_{i}\left(f_{i+1}-l_{i}\right)+\left(f_{i+1}-l_{i}\right)-h_{i} d_{i+1}-k_{i} .
\end{aligned}
$$

The interpolating curve lies above the given straight line $y=$ $m x+c$ if $U_{i}(\theta)>0$ because $Q_{i}(\theta)>0$ for all $\alpha_{i} \geq 0, i=$ $0,1, \ldots, n-1$. The necessary conditions are $f_{i}-k_{i}>0$ and $f_{i+1}-l_{i}>0$, respectively. Thus the sufficient condition for the interpolating curve to lie above the given straight line can be derived from $C_{i 1}>0$ and $C_{i 2}>0$ :

$$
\begin{aligned}
& C_{i 1}>0 \Longrightarrow \\
& f_{i}+\alpha_{i}\left(f_{i}-k_{i}\right)+\left(f_{i}-k_{i}\right)+h_{i} d_{i}-l_{i}>0, \\
& C_{i 2}>0 \Longrightarrow \\
& f_{i+1}+\alpha_{i}\left(f_{i+1}-l_{i}\right)+\left(f_{i+1}-l_{i}\right)-h_{i} d_{i+1}-k_{i}>0 .
\end{aligned}
$$

Inequalities (28) provide the following:

$$
\begin{gathered}
\alpha_{i}>\frac{-h_{i} d_{i}+l_{i}-f_{i}}{f_{i}-k_{i}}, \\
\alpha_{i}>\frac{h_{i} d_{i+1}+k_{i}-f_{i+1}}{f_{i+1}-l_{i}} .
\end{gathered}
$$

For $i=0,1, \ldots, n-1$, conditions in (29) and (30) can be combined to form the following sufficient condition:

$$
\alpha_{i}>\max \left\{0, \frac{-h_{i} d_{i}+l_{i}-f_{i}}{f_{i}-k_{i}}, \frac{h_{i} d_{i+1}+k_{i}-f_{i+1}}{f_{i+1}-l_{i}}\right\} .
$$

The above result is stated in Theorem 3 below.

Theorem 3. The rational cubic Ball interpolant $S_{i}(x)$ lies above the given straight line $y=m x+c$, if in subinterval $\left[x_{i}, x_{i+1}\right]$, $i=0,1, \ldots, n-1$, the parameter satisfy the condition

$$
\begin{aligned}
\alpha_{i}= & \gamma_{i} \\
& +\max \left\{0, \frac{-h_{i} d_{i}+l_{i}-f_{i}}{f_{i}-k_{i}}, \frac{h_{i} d_{i+1}+k_{i}-f_{i+1}}{f_{i+1}-l_{i}}\right\},
\end{aligned}
$$


2.7. Monotonicity Preserving. There are two cases for strictly monotonic preserving interpolation.

Case 1 (if $\Delta_{i}=0, i=0,1, \ldots, n-1$ ). If $\Delta_{i}=0$, that is, some data are collinear or comonotone, then the rational cubic Ball interpolant defined by (1) is constant on the subinterval $\left[x_{i}, x_{i+1}\right], i=0,1, \ldots, n-1$. This can be achieved by setting the first derivative $d_{i}=d_{i+1}=0$, such that $S_{i}(x)=f_{i}=f_{i+1}$.

Case 2 (if $\Delta_{i}>0, i=0,1, \ldots, n-1$ ). For this case, we have the following theorem.

Theorem 4. The rational cubic Ball interpolation defined in (1) preserves the monotonic increasing data on each subinterval $\left[x_{i}, x_{i+1}\right], i=0,1, \ldots, n-1$ if the parameter satisfies the following condition:

$$
\alpha_{i}>\frac{d_{i}+d_{i+1}}{\Delta_{i}} .
$$

Proof. Shape preserving interpolation for strictly monotonic data can be stated as follows.

For monotonic increasing data $\left\{\left(x_{i}, f_{i}\right), i=0,1, \ldots, n\right\}$ with $x_{0}<x_{1}<\cdots<x_{n}$ and satisfying $f_{0}<f_{1}<\cdots<f_{n}$, we want to construct the monotonic interpolating curve by using the rational cubic Ball defined by (1). The rational cubic Ball $S(x)$ will produce the monotonic increasing interpolating curve if and only if the first derivative at the knot $x_{i}, i=$ $0,1, \ldots, n$ satisfies

$$
S^{(1)}(x)>0
$$

The first derivative $S^{(1)}(x)$ is equal to

$$
S^{(1)}(x)=\frac{\sum_{j=0}^{4} A_{i j}(1-\theta)^{4-j} \theta^{j}}{\left[Q_{i}(\theta)\right]^{2}}
$$

with

$$
\begin{aligned}
& A_{i 0}=d_{i} \\
& A_{i 1}=2\left(\alpha_{i}+3\right) \Delta_{i}-2 d_{i+1}, \\
& A_{i 2}=12 \Delta_{i}+3 \alpha_{i} \Delta_{i}+\left(\alpha_{i}+3\right)\left(\alpha_{i} \Delta_{i}-d_{i}-d_{i+1}\right), \\
& A_{i 3}=2\left(\alpha_{i}+3\right) \Delta_{i}-2 d_{i} \\
& A_{i 4}=d_{i+1} .
\end{aligned}
$$

The following are the necessary conditions to obtain monotonicity increasing interpolant:

$$
\begin{array}{ll}
d_{i} \geq 0 & \text { for } i=0,1, \ldots, n, \\
\Delta_{i}>0 & \text { for } i=0,1, \ldots, n-1
\end{array}
$$

The sufficient condition for the monotonicity of the rational cubic Ball $S(x)$ can be derived from (34). The first derivative $S^{(1)}(x)>0$ if and only if $A_{i j} \geq 0, j=0,1,2,3,4$. Clearly
$A_{i 0} \geq 0$ and $A_{i 3} \geq 0$. The remaining inequalities $A_{i j} \geq 0$, $j=1,2,3$ give us

$$
\begin{aligned}
2\left(\alpha_{i}+3\right) \Delta_{i}-2 d_{i+1} & >0, \\
\alpha_{i} \Delta_{i}-d_{i}-d_{i+1} & >0, \\
\left(\alpha_{i}+3\right) \Delta_{i}-d_{i} & >0 .
\end{aligned}
$$

The sufficient condition for monotonicity of the rational cubic Ball interpolant is derived as follows.

From (38), we obtain

$$
\alpha_{i}>\frac{d_{i+1}}{\Delta_{i}} .
$$

Similarly (39) and (40) lead to the inequalities (42):

$$
\begin{aligned}
& \alpha_{i}>\frac{d_{i}+d_{i+1}}{\Delta_{i}}, \\
& \alpha_{i}>\frac{d_{i}}{\Delta_{i}} .
\end{aligned}
$$

Combining conditions (41), (42), and (43) gives the sufficient condition for the monotonicity preserving of the rational cubic Ball interpolation.

$$
\alpha_{i}>\frac{d_{i}+d_{i+1}}{\Delta_{i}} .
$$

This completes the proof for Theorem 4 .

Condition in (44) can be rewritten as

$$
\alpha_{i}=\mu_{i}+\frac{d_{i}+d_{i+1}}{\Delta_{i}}
$$

with $0<\mu_{i} \leq 0.25$

2.8. Convexity Preserving. We assume that a strictly convex data set $\left\{\left(x_{i}, f_{i}\right), i=0,1, \ldots, n\right\}$ is given with $x_{0}<x_{1}<\cdots<$ $x_{n}$ such that $\Delta_{0}<\Delta_{1}<\cdots<\Delta_{i-1}<\Delta_{i}<\cdots<\Delta_{n-1}$, and

$$
d_{0}<\Delta_{0}<d_{1}<\Delta_{1}<\cdots<\Delta_{n-2}<\cdots<d_{n}<\Delta_{n-1} .
$$

We want to produce the convex interpolating curve on each subinterval $\left[x_{i}, x_{i+1}\right], i=0,1, \ldots, n-1$ by using rational cubic Ball defined by (1). The function is convex if $S^{(2)}(x)>0$. After some simplification, the second derivative of the rational cubic Ball $S^{(2)}(x)$ is given as

$$
S^{(2)}(x)=\frac{\sum_{j=0}^{3} E_{i j}(1-\theta)^{3-j} \theta^{j}}{h_{i}\left[Q_{i}(\theta)\right]}
$$

with

$$
\begin{aligned}
& E_{i 0}=2\left[\left(\alpha_{i}+2\right)\left(\Delta_{i}-d_{i}\right)-\left(d_{i+1}-\Delta_{i}\right)\right], \\
& E_{i 1}=6\left(\Delta_{i}-d_{i}\right), \\
& E_{i 2}=6\left(d_{i+1}-\Delta_{i}\right), \\
& E_{i 3}=2\left[\left(\alpha_{i}+2\right)\left(d_{i+1}-\Delta_{i}\right)-\left(\Delta_{i}-d_{i}\right)\right] .
\end{aligned}
$$

In order to derive the sufficient condition for convexity preserving, there are two cases that need to be considered. 
TABle 2: Positive data from Hussain et al. [8].

\begin{tabular}{ccccc}
\hline$i$ & 0 & 1 & 2 & 3 \\
\hline$x_{i}$ & 0 & 1 & 1.7 & 1.8 \\
$f_{i}$ & 0.25 & 1 & 11.10 & 25 \\
$d_{i}$ & -7.2962 & 8.7962 & 123.429 & 154.571 \\
\hline
\end{tabular}

Case 1. If the convex data satisfy $d_{i+1}-\Delta_{i}=0$ and $\Delta_{i}-d_{i}=0$, then the rational cubic Ball interpolant will produce a linear segment on the subinterval $\left[x_{i}, x_{i+1}\right], i=0,1, \ldots, n-1$ such that

$$
S_{i}(x)=(1-\theta) f_{i}+\theta f_{i+1} .
$$

Case 2. If the convex data satisfy (46), then the necessary condition for convexity is $d_{i+1}-\Delta_{i}>0$ and $\Delta_{i}-d_{i}>0$. Thus $S^{(2)}(x)>0$ if and only if $E_{i 0}>0$ and $E_{i 3}>0$. Both conditions provide the following inequalities:

$$
\begin{aligned}
& 2\left[\left(\alpha_{i}+2\right)\left(\Delta_{i}-d_{i}\right)-\left(d_{i+1}-\Delta_{i}\right)\right]>0 \\
& 2\left[\left(\alpha_{i}+2\right)\left(d_{i+1}-\Delta_{i}\right)-\left(\Delta_{i}-d_{i}\right)\right]>0 .
\end{aligned}
$$

Simplifying conditions (50) lead to

$$
\begin{gathered}
\alpha_{i}>\frac{d_{i+1}-\Delta_{i}}{\Delta_{i}-d_{i}}, \\
\alpha_{i}>\frac{\Delta_{i}-d_{i}}{d_{i+1}-\Delta_{i}} .
\end{gathered}
$$

These two conditions give the sufficient condition for the convexity of the rational cubic Ball interpolant

$$
\alpha_{i}>\max \left\{\frac{d_{i+1}-\Delta_{i}}{\Delta_{i}-d_{i}}, \frac{\Delta_{i}-d_{i}}{d_{i+1}-\Delta_{i}}\right\} .
$$

The result is summarized as Theorem 1 below.

Theorem 1. The rational cubic Ball defined by (1) preserves the convexity of the data if the parameter satisfies the following condition:

$$
\alpha_{i}=\eta_{i}+\max \left\{\frac{d_{i+1}-\Delta_{i}}{\Delta_{i}-d_{i}}, \frac{\Delta_{i}-d_{i}}{d_{i+1}-\Delta_{i}}\right\}
$$

with $0<\eta_{i} \leq 0.25$.

\section{Results and Discussion}

This section is devoted to the numerical results for the constrained data interpolation subject to constraint line above straight line, monotonicity preservation and convexity preservation.

3.1. Numerical Example 2. The positive data sets from Hussain et al. [8] are listed in Table 2.

For this example, Figure 2(a) shows the cubic Ball polynomial with $\alpha_{i}=0, i=0,1,2$. Meanwhile Figures 2(b) and 2(c) show the positive interpolating curve after applying the result from Theorem 1 with $\lambda_{i}, i=0,1,2$, varying as indicated in both Figures 2(b) and 2(c).

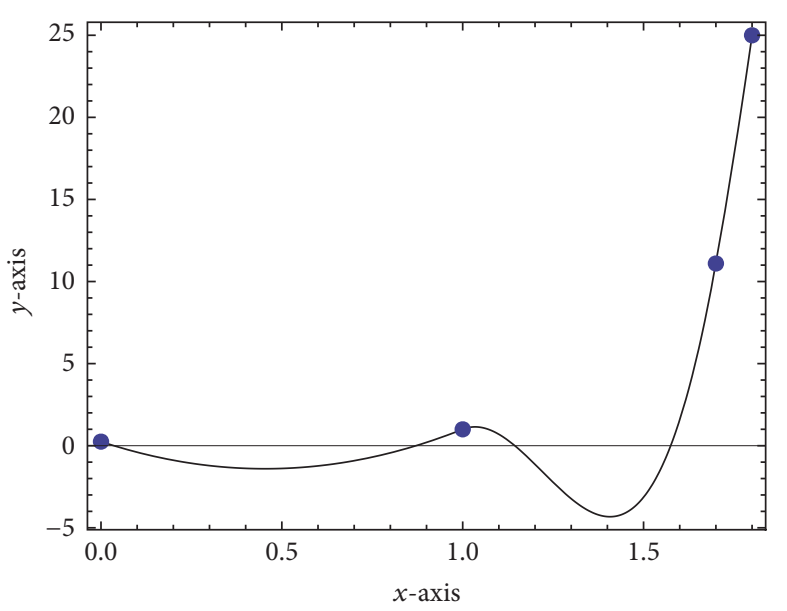

(a) $\alpha_{i}=0$

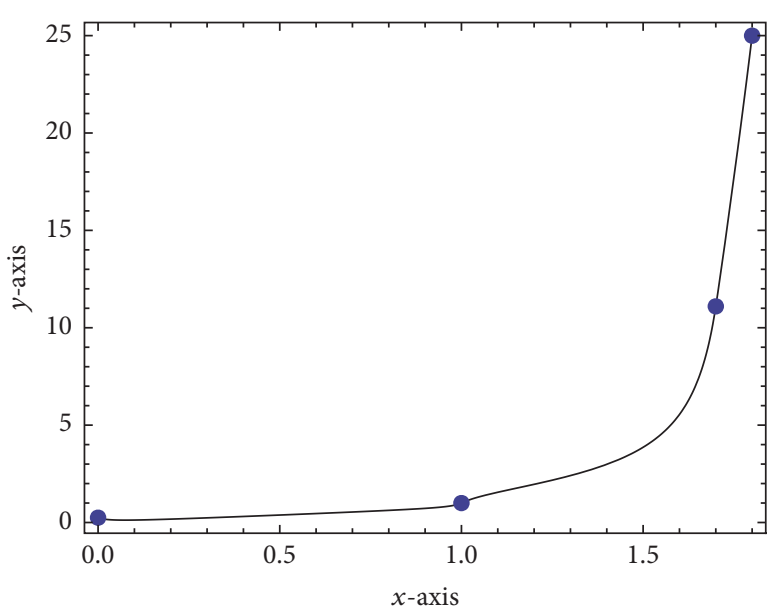

(b) $\lambda_{i}=0.1$

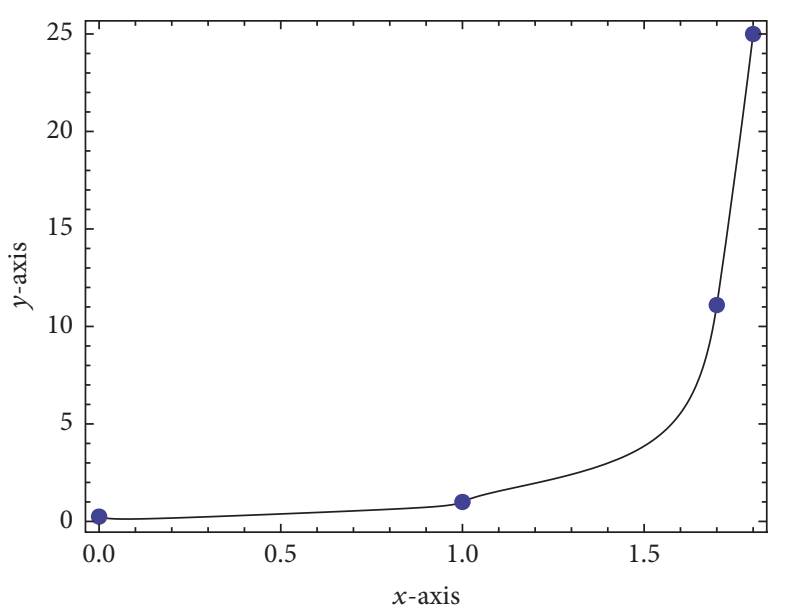

(c) $\lambda_{i}=0.25$

FIGURE 2: Positivity preserving using the proposed rational cubic Ball.

3.2. Numerical Example 3. The data in Table 3 lie above the straight line $y=0.2 x+0.1$.

Figure 3(a) shows that the cubic Ball cannot produce the interpolating curve that lies above the given straight line. 


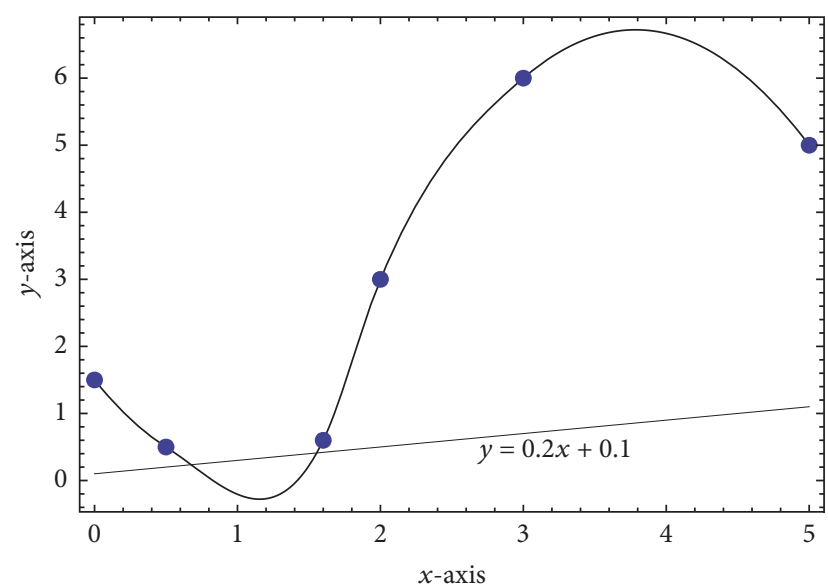

(a) $\alpha_{i}=0$

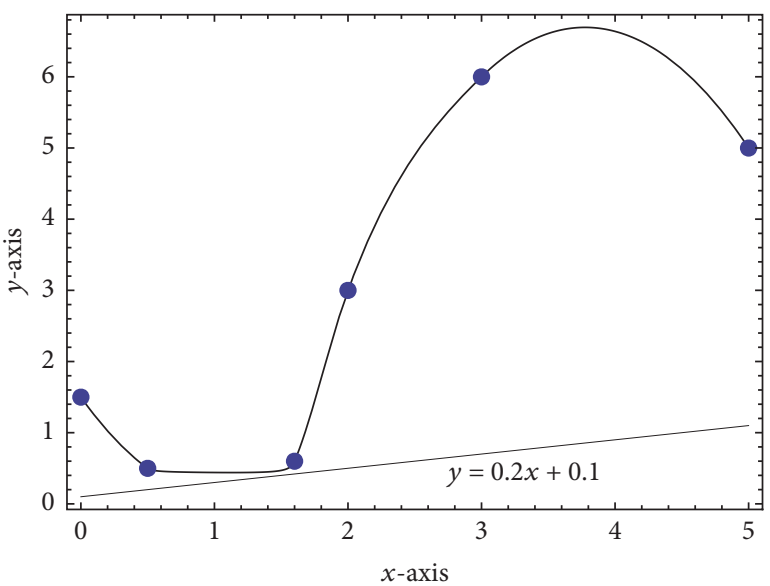

(b) $\gamma_{i}=0.1$

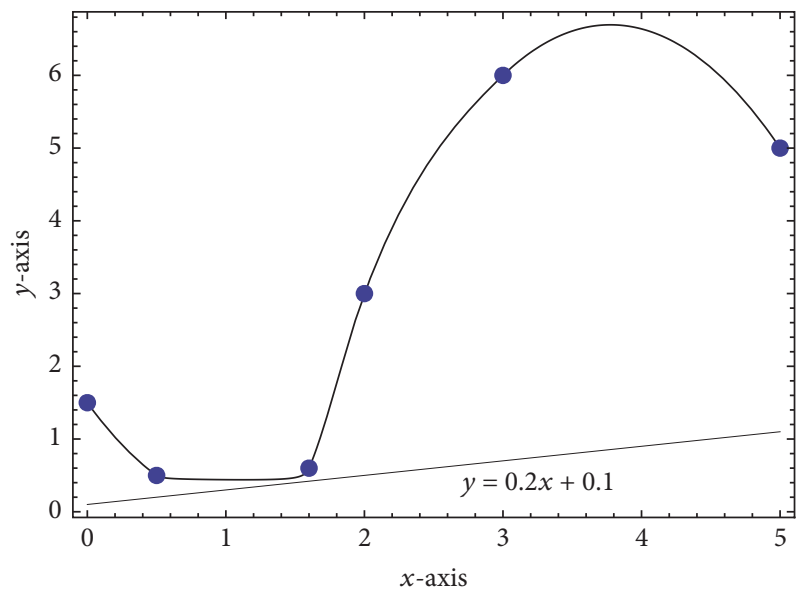

(c) $\gamma_{i}=0.25$

FIGURE 3: Constraint interpolation for data in Table 3.

Applying Theorem 3, we obtain the interpolating curve that lies above a straight line with $\gamma_{i}=0.1$ for Figure 3(b) and $\gamma_{i}=0.1$ for Figure 3(c).

3.3. Numerical Example 4. Table 4 shows the monotone data taken from Akima [10].
TABLE 3: Constraint data.

\begin{tabular}{ccccccc}
\hline$i$ & 0 & 1 & 2 & 3 & 4 & 5 \\
\hline$x_{i}$ & 0 & 0.5 & 1.6 & 2 & 3 & 5 \\
$f_{i}$ & 2 & 0.6 & 0.33 & 0.35 & 1 & 0.5 \\
$d_{i}$ & -2.653 & -1.357 & 4.424 & 5.143 & 1.833 & -2.833 \\
\hline
\end{tabular}

Figure 4(a) shows the cubic Ball cannot preserve the monotonicity of the data set. This flaw has been recovered nicely by using condition (45), Figure 4(b) with $\mu_{i}=0.1$ and Figure 4 (c) with $\mu_{i}=0.25$. Clearly the resulting monotonic interpolating curve is visually pleasing.

3.4. Numerical Example 5. Table 5 shows the convex data from Brodlie and Butt [7]

Figure 5(a) shows the cubic Ball cannot produce the convex interpolating curve. Condition (52) guarantees to produce convex interpolating curve on the whole interval. This can be verified by plotting the second derivative graph for Figures 5(a) and 5(b), respectively. From Figure 6(a) the second derivative for cubic Ball polynomial is a straight line and has some negative value which contradicts with the sufficient condition given in (49) and (54). Figure 6(b) shows that the proposed rational cubic Ball interpolant produces the convex interpolating curve since its second derivative is greater than zero.

3.5. Application in Image Processing. An application of the proposed rational cubic Ball interpolation in image processing is discussed in this section. Firstly the univariate spline given in (1) is extended to the bivariate cases by using tensor product approach. The rational bicubic function over each rectangular patch $\left[x_{i}, x_{i+1}\right] \times\left[y_{j}, y_{j+1}\right], i=0,1, \ldots, n-1$; $j=0,1, \ldots, m-1$ is defined as follows:

$$
S_{i, j}(x, y)=A_{i}(\theta) F_{i, j} A_{j}(\phi)^{T},
$$

where

$$
\begin{aligned}
& F_{i, j}=\left[\begin{array}{cccc}
F_{i, j} & F_{i, j+1} & F_{i, j}^{y} & F_{i, j+1}^{y} \\
F_{i+1, j} & F_{i+1, j+1} & F_{i+1, j}^{y} & F_{i+1, j+1}^{y} \\
F_{i, j}^{x} & F_{i, j+1}^{x} & F_{i, j}^{x y} & F_{i, j+1}^{x y} \\
F_{i+1, j}^{x} & F_{i+1, j+1}^{x} & F_{i+1, j}^{x y} & F_{i+1, j+1}^{x y}
\end{array}\right], \\
& A_{i}(\theta)=\left[\begin{array}{llll}
a_{0}(\theta) & a_{1}(\theta) & a_{2}(\theta) & a_{3}(\theta)
\end{array}\right], \\
& A_{j}(\phi)=\left[\begin{array}{llll}
\widehat{a}_{0}(\phi) & \widehat{a}_{1}(\phi) & \widehat{a}_{2}(\phi) & \widehat{a}_{3}(\phi)
\end{array}\right],
\end{aligned}
$$

where

$$
\begin{aligned}
& a_{0}(\theta)=\frac{(1-\theta)^{2}+\left(\alpha_{i, j}+2\right)(1-\theta)^{2} \theta}{q_{i}(\theta)}, \\
& a_{1}(\theta)=\frac{\theta^{2}+\left(\alpha_{i, j}+2\right)(1-\theta) \theta^{2}}{q_{i}(\theta)}, \\
& a_{2}(\theta)=\frac{h_{i}(1-\theta)^{2} \theta}{q_{i}(\theta)},
\end{aligned}
$$


TABLE 4: Monotone data from [10].

\begin{tabular}{ccccccccccccc}
\hline$i$ & 0 & 1 & 2 & 3 & 4 & 5 & 6 & 7 & 8 & 9 & 10 \\
\hline$x_{i}$ & 0 & 2 & 3 & 5 & 6 & 8 & 9 & 11 & 12 & 14 & 15 \\
$f_{i}$ & 10 & 10 & 10 & 10 & 10 & 10 & 10.5 & 15 & 50 & 60 & 85 \\
$d_{i}$ & 0 & 0 & 0 & 0 & 0 & 0 & 1.0833 & 24.0833 & 25 & 18.3333 & 31.6667 \\
\hline
\end{tabular}

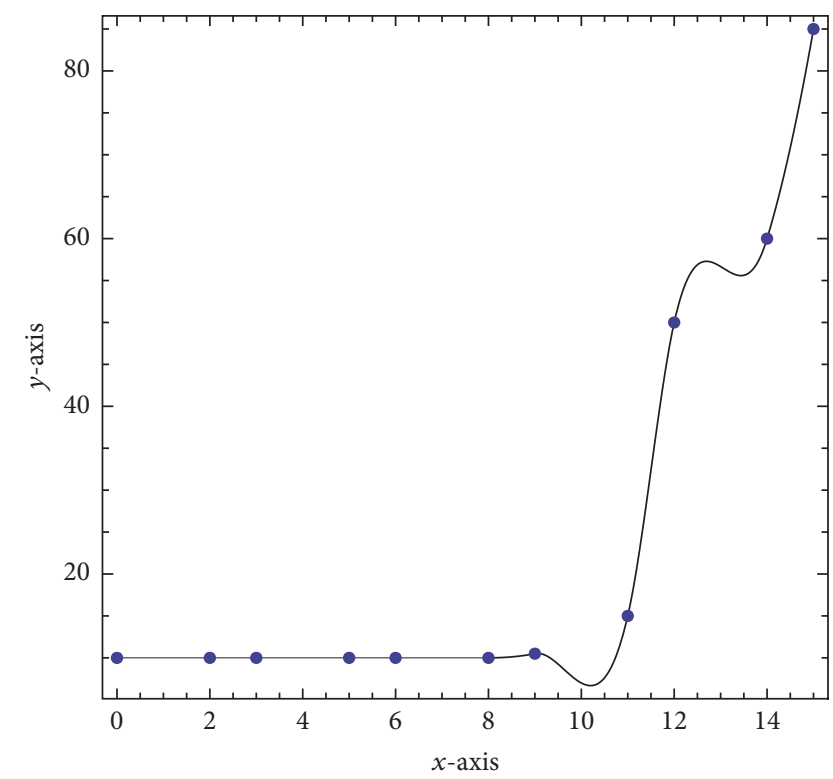

(a) $\alpha_{i}=0$

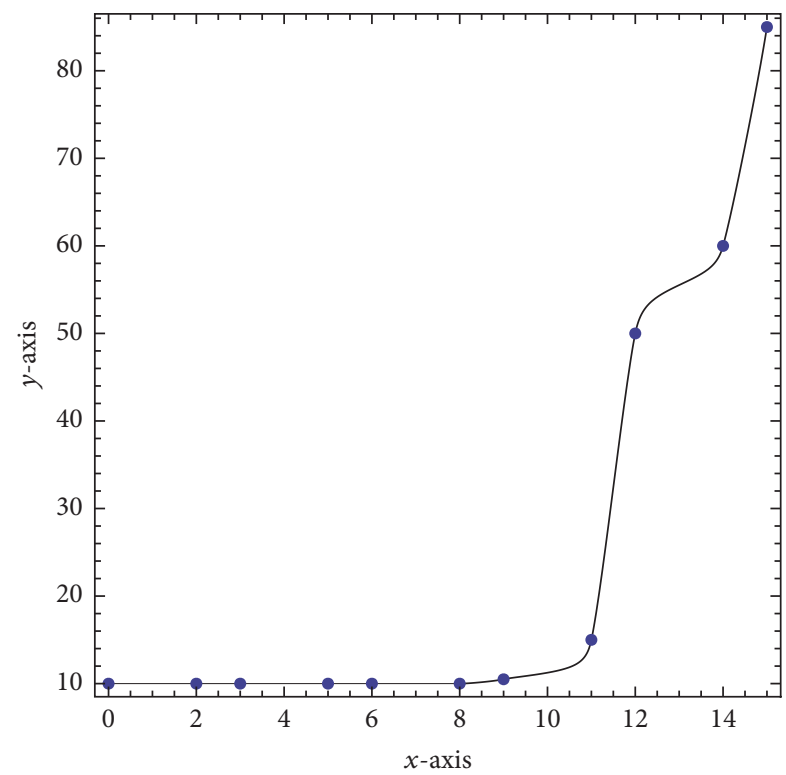

(b) $\mu_{i}=0.1$

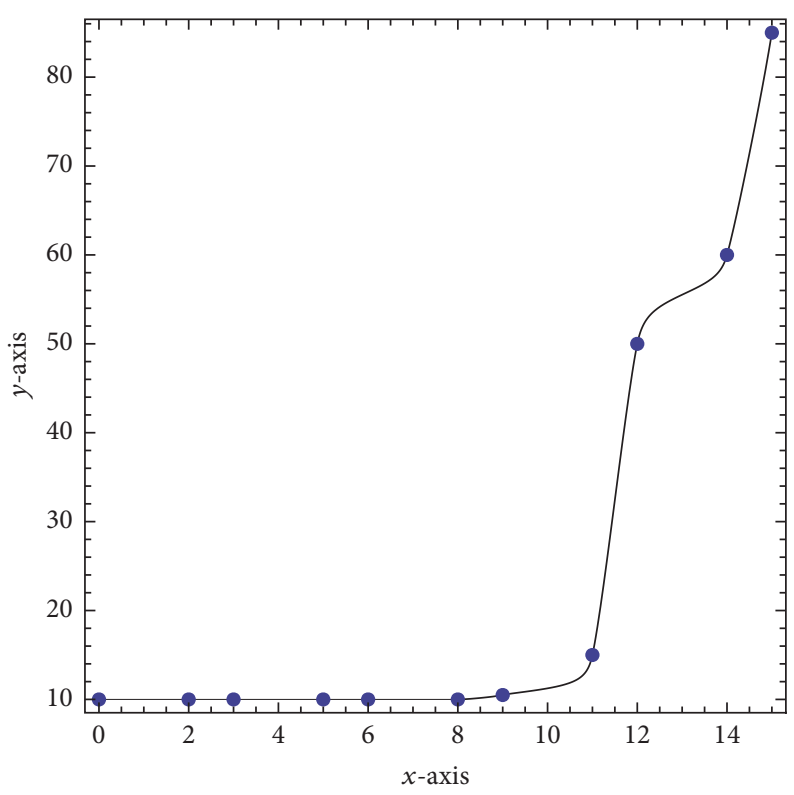

(c) $\mu_{i}=0.25$

FIgURE 4: Interpolating curves for data in Table 4.

$$
\begin{aligned}
& a_{3}(\theta)=-\frac{h_{i}(1-\theta) \theta^{2}}{q_{i}(\theta)}, \\
& \widehat{a}_{0}(\phi)=\frac{(1-\phi)^{2}+\left(\widehat{\alpha}_{i, j}+2\right)(1-\phi)^{2} \phi}{q_{j}(\phi)},
\end{aligned}
$$

$$
\begin{aligned}
& \widehat{a}_{1}(\phi)=\frac{\phi^{2}+\left(\widehat{\alpha}_{i, j}+2\right)(1-\phi) \phi^{2}}{q_{j}(\phi)}, \\
& \widehat{a}_{2}(\phi)=\frac{\widehat{h}_{j}(1-\phi)^{2} \phi}{q_{j}(\phi)},
\end{aligned}
$$




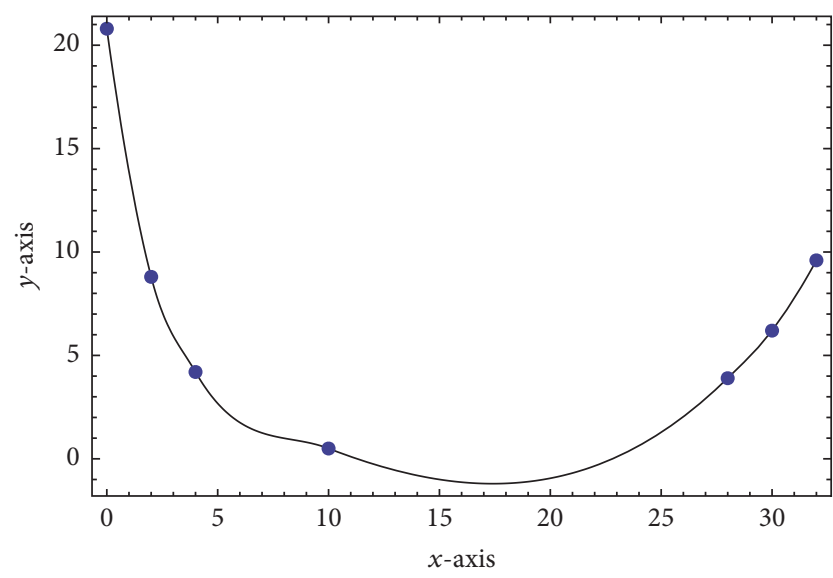

(a) $\alpha_{i}=0$

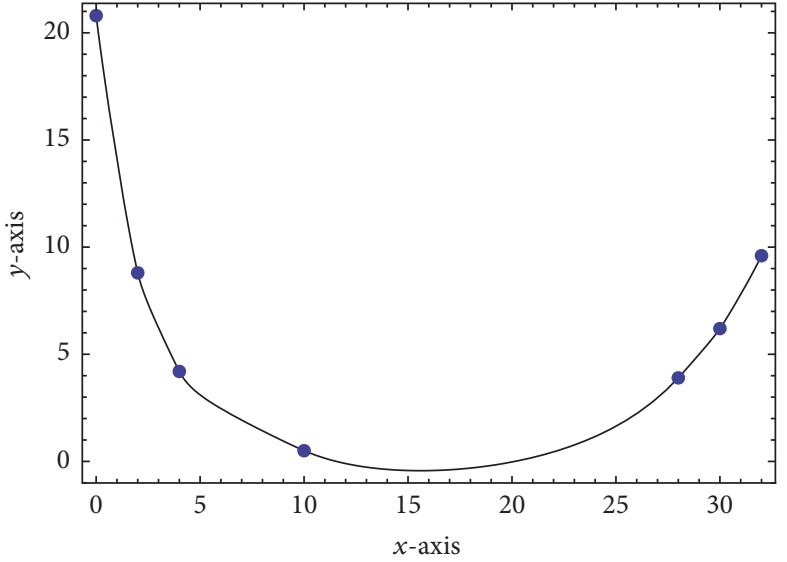

(b) $\eta_{i}=0.1$

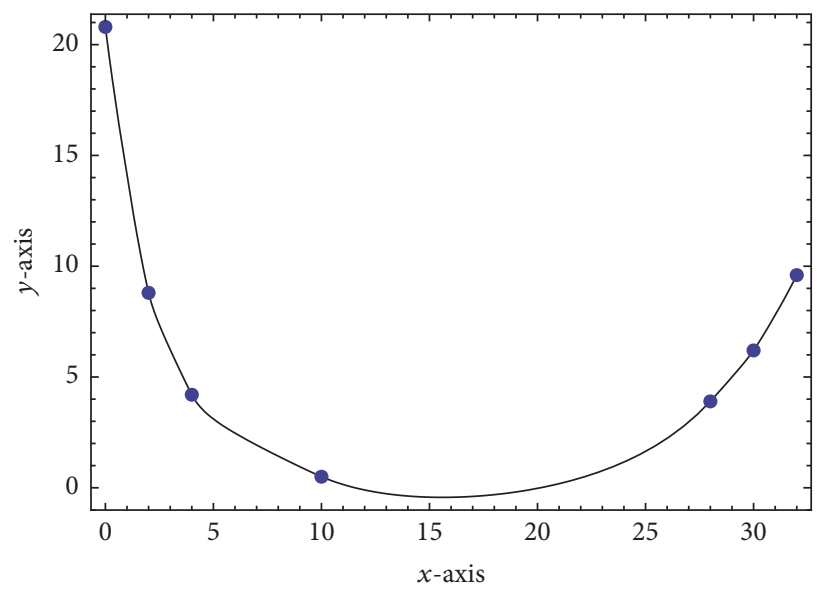

(c) $\eta_{i}=0.25$

FIGURE 5: Interpolating curve for convex data set.

TABle 5: Convex data from [7].

\begin{tabular}{cccccccc}
\hline$i$ & 1 & 2 & 3 & 4 & 5 & 6 & 7 \\
\hline$x_{i}$ & 0 & 2 & 4 & 10 & 28 & 30 & 32 \\
$f_{i}$ & 20.8 & 8.8 & 4.2 & 0.5 & 3.9 & 6.2 & 9.6 \\
$d_{i}$ & -7.85 & -4.15 & -1.8792 & -0.4153 & 1.0539 & 1.425 & 1.975 \\
\hline
\end{tabular}

$$
\begin{aligned}
\widehat{a}_{3}(\theta) & =-\frac{\widehat{h}_{j}(1-\phi) \phi^{2}}{q_{j}(\phi)}, \\
\theta & =\frac{x-x_{i}}{h_{i}}, \\
\phi & =\frac{y-y_{j}}{\widehat{h}_{j}}, \\
h_{i} & =x_{i+1}-x_{i}, \\
\widehat{h}_{j} & =y_{j+1}-y_{j},
\end{aligned}
$$

$$
\begin{array}{cc}
q_{i}(\theta)=1+\alpha_{i, j} \theta(1-\theta), & \alpha_{i, j} \geq 0, \\
q_{j}(\phi)=1+\widehat{\alpha}_{i, j} \phi(1-\phi), & \widehat{\alpha}_{i, j} \geq 0,
\end{array}
$$

where $F_{i, j}^{x}$ and $F_{i, j}^{y}$ are partial derivatives on $x$ and $y$ directions, respectively. Meanwhile $F_{i, j}^{x y}$ is mixed partial derivatives (twists) at the interior points [12]. All can be estimated by using the method discussed in [12].

We apply the rational bicubic Ball function to image interpolation application. The main spotlight here is the image upscaling. Given the original image $256 \times 256$, then we can upscale the image with factor two or four and so on. To achieve this, we employ the following algorithm.

Algorithm 3.

Input. Image $m$ by $n$ pixel and parameter values $\alpha_{i, j}$ and $\widehat{\alpha}_{i, j}$. Output. Upscaling image, computation time, and PSNR value. 


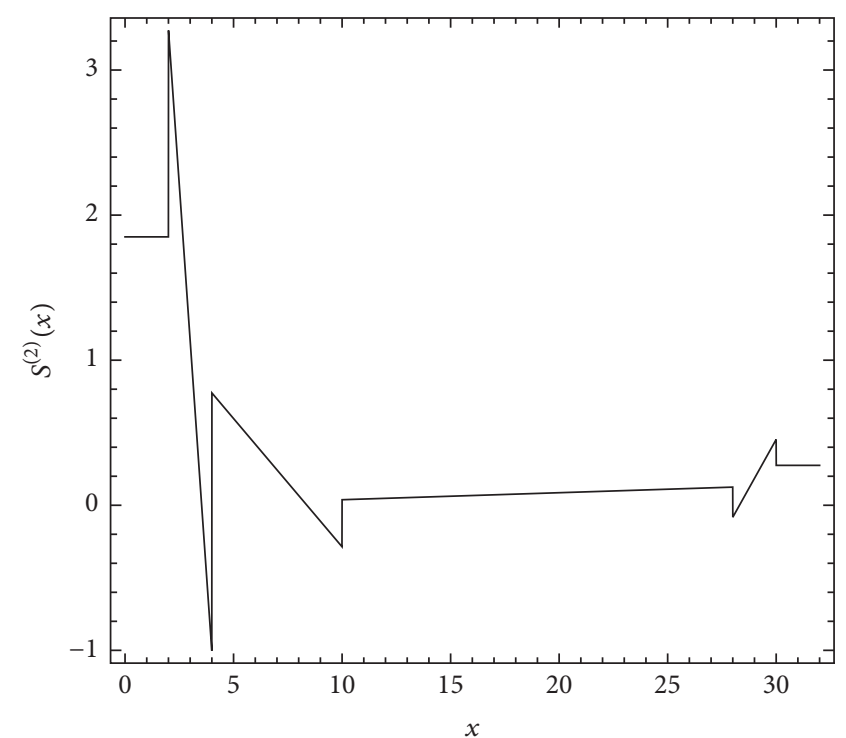

(a) Cubic Ball

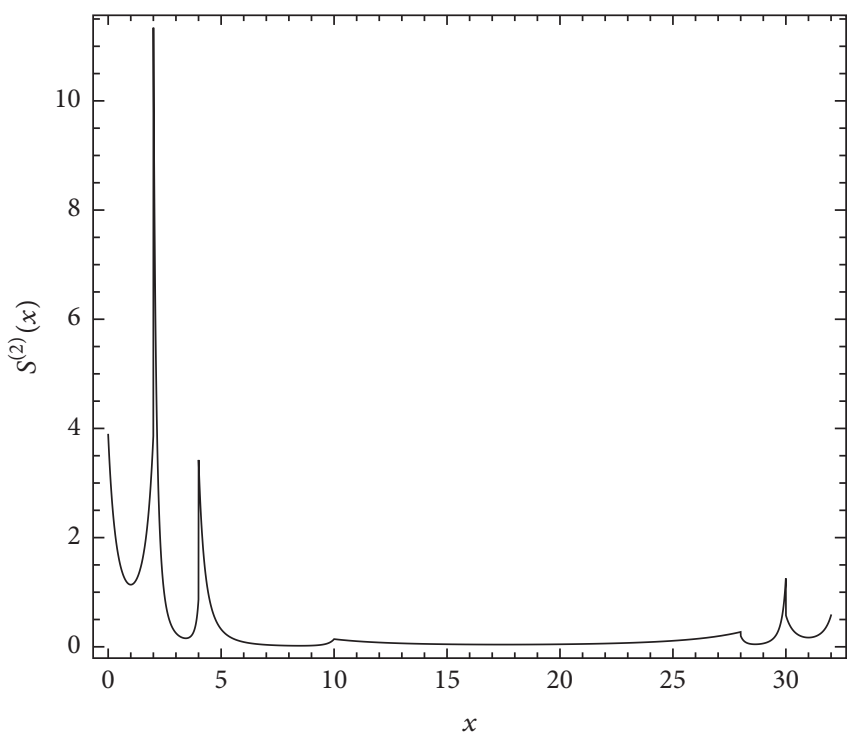

(b) The proposed scheme

FIgURE 6: Second derivative plot for Figures 5(a) and 5(b).

Step 1. Let $(i, j), i=1,2, \ldots, m, j=1,2, \ldots, n$ represent input pixel indexes and $z_{i j}(0-255)$ be their corresponding grayscale intensity. Our aim is to find the function; that is, $\bar{z}_{i j}=F(i, j)$ which interpolate the given input pixels.

Step 2. Construct rectangular mesh for given input pixels using our rational bicubic Ball surface defined by (55).

Step 3. Let

arbitrary input pixel be $(x, y)$;

$\left(x^{\prime}, y^{\prime}\right)$ be arbitrary output pixel;

$k$ be scaling factor at pixel $(1,1)(k=2$ or $k=4)$.

Output Image. $m k$ by $n k$ pixels will be obtained by the following transformation:

$$
\begin{aligned}
\left(\begin{array}{c}
x^{\prime} \\
y^{\prime}
\end{array}\right)= & \left(\begin{array}{cc}
\frac{m k-1}{m-1} & 0 \\
0 & \frac{n k-1}{n-1}
\end{array}\right)\left(\begin{array}{l}
x \\
y
\end{array}\right) \\
& +\left(\begin{array}{c}
\frac{m(1-k)}{m-1} \\
\frac{n(1-k)}{n-1}
\end{array}\right) .
\end{aligned}
$$

Step 4. Identify the rectangles with the missing grayscale intensity values.

The original pixels and their intensity values of input image are at vertex of rectangular mesh.

Step 5. Estimate the derivative at each vertex of rectangular (input pixels) by method discussed in [12].
Step 6. Estimate missing grayscale intensity value in Step 4 by using the proposed rational cubic Ball scheme defined in (55).

Step 7. Display the result and compare the performance of proposed method against nearest neighbor, bilinear interpolation, and bicubic spline interpolation by using peak signal to noise ratio (PSNR).

We test the proposed rational bicubic Ball functions for image upscaling by using five different images shown in Figure 7. We compare the performance with nearest neighbor and bilinear and bicubic spline interpolation. Table 6 summarized the results including the value of PSNR. The value of PSNR will reflect the quality of image that is the larger PSNR value the higher quality of images [13]. It is measured in decibels $(\mathrm{dB})$.

Peak signal to noise ratio (PSNR) is defined as

$$
\mathrm{PSNR}=10 \log _{10}\left(\frac{255^{2}}{\mathrm{MSE}}\right)
$$

where MSE is mean square error given as

$$
\mathrm{MSE}=\frac{1}{m n} \sum_{i=0}^{m} \sum_{j=0}^{n}\left|z_{i j}-\bar{z}_{i j}\right|^{2}
$$

with $z_{i j}$ and $\bar{z}_{i j}$ defined as in Step 2 in Algorithm 3.

From all PSNR value, we conclude that the proposed scheme is at par with the standard scheme for image interpolation, that is, bicubic spline. In fact, for some images, the proposed scheme gives higher PSNR compared with bicubic spline interpolation. To obtain the result we use parameter value as $\alpha_{i j}=\widehat{\alpha}_{i j}=0.1$. Finally, for all tested images, the proposed rational bicubic Ball surface gives less computation 

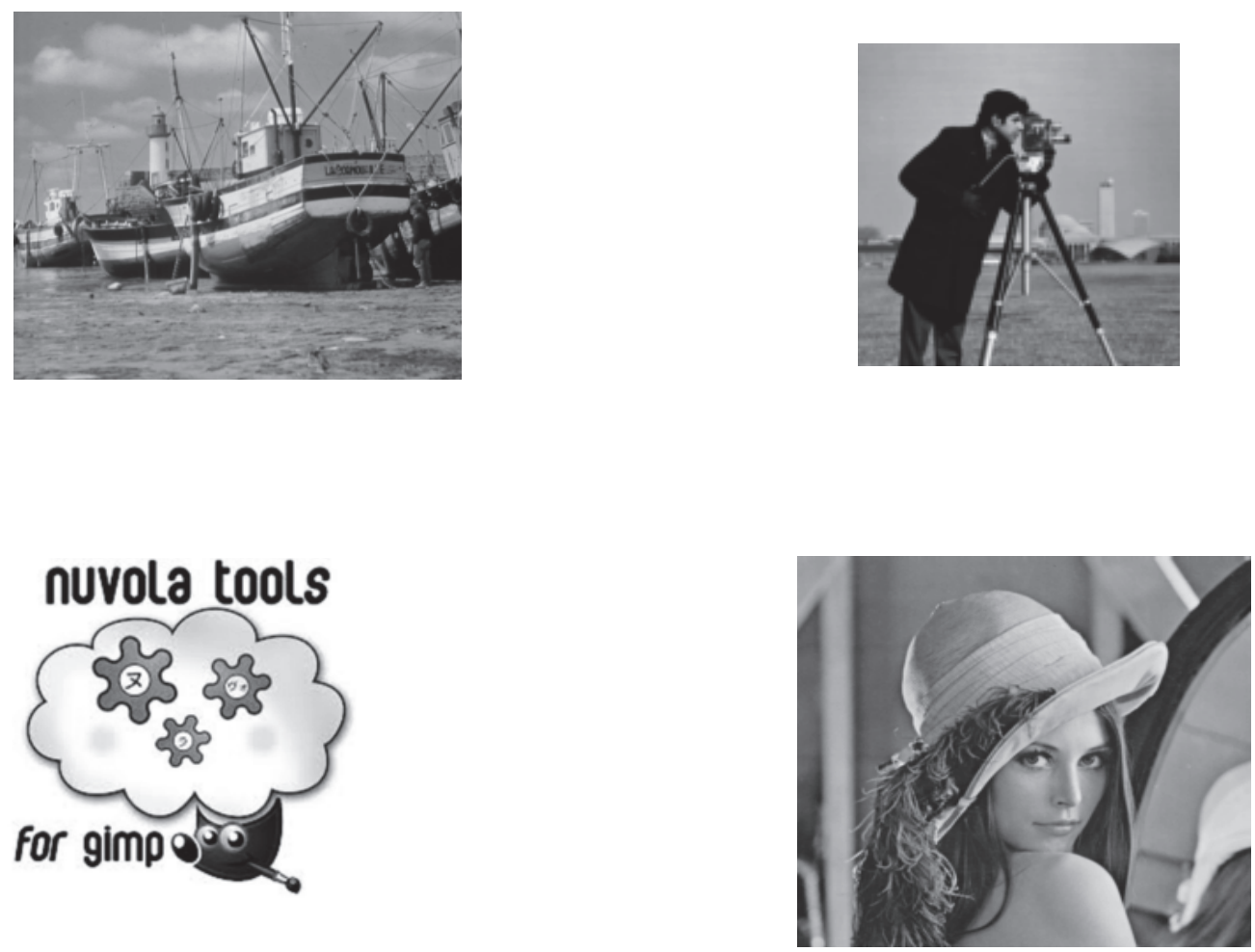

FIgURE 7: Tested images: boat, cameraman, nuvola, lena, and thumb print.

TABLE 6: PSNR value for image upscaling.

\begin{tabular}{lccccc}
\hline Method/images & Boat & Cameraman & Nuvola & Lena & Thumb print \\
\hline Nearest neighbor & 33.85 & 39.42 & 35.94 & 36.10 & 35.40 \\
Bilinear interpolation & 34.02 & 39.99 & 36.30 & 36.18 & 35.75 \\
Bicubic spline interpolation & $\mathbf{3 4 . 4 6}$ & 39.86 & 36.73 & $\mathbf{3 7 . 0 0}$ & 35.76 \\
Proposed method & 34.45 & $\mathbf{4 0 . 0 8}$ & $\mathbf{3 6 . 7 5}$ & 36.64 & $\mathbf{3 5 . 8 2}$ \\
\hline
\end{tabular}

TABle 7: Computation time.

\begin{tabular}{lccccc}
\hline Method/images & Boat & Cameraman & Nuvola & Lena & Thumb print \\
\hline Bicubic spline interpolation & 0.6340 & 0.5273 & 0.5492 & 0.5278 & 0.2245 \\
The proposed method & $\mathbf{0 . 6 2 6 8}$ & $\mathbf{0 . 5 1 4 8}$ & $\mathbf{0 . 5 3 8 9}$ & $\mathbf{0 . 5 0 5 4}$ & $\mathbf{0 . 2 1 1 3}$ \\
\hline
\end{tabular}


time comparing with bicubic spline interpolation. Computation time is calculated based on CPU time to generate the upscaling images with factor 4 .

\section{Conclusion}

This study has proposed new rational bicubic Ball function with one parameter. This rational cubic has been used for shape preserving interpolation, that is, positivity, monotonicity, convexity, and constrained data interpolation. The univariate rational cubic Ball is extended to the bivariate cases. The rational bicubic Ball function is used for image upscaling problem in image processing. From all results, we conclude that the proposed scheme is at par with standard scheme, that is, bicubic spline interpolation. Further works can be explored such as to determine the optimum value of the parameters $\alpha_{i j}$ and $\widehat{\alpha}_{i j}$ for image processing application. This can be achieved by using genetic algorithm (GA) or neural network (NN) algorithm such as in Hussain et al. [14]. Furthermore, we intend to apply the proposed scheme for image resizing and image zooming in medical imaging. The proposed rational cubic Ball can also is extended to $C^{2}$ continuous interpolating curve in line with the work of Karim and Kong [15].

\section{Conflicts of Interest}

The authors declare that there are no conflicts of interest regarding the publication of this paper.

\section{Authors' Contributions}

Both authors give equal contribution throughout the preparation of the paper.

\section{Acknowledgments}

This research is fully supported by Universiti Teknologi PETRONAS (UTP) through a research Grant YUTP: 0153AAH24 (Spline Triangulation for Spatial Interpolation of Geophysical Data). Special thanks are due to staffs at Research Management Centre (RMC), UTP.

\section{References}

[1] S. Abbas, M. Z. Hussain, and M. Irshad, "GA based rational cubic B-spline representation for still image interpolation," Pakistan Journal of Statistics and Operation Research, vol. 12, no. 4, pp. 753-763, 2016.

[2] S. Abbas, M. Z. Hussain, and M. Irshad, "Image interpolation by rational ball cubic B-spline representation and genetic algorithm," Alexandria Engineering Journal, 2016.

[3] A. Majeed, A. R. M. Piah, R. U. Gobithaasan, and Z. R. Yahya, "Craniofacial reconstruction using rational cubic ball curves," PLoS ONE, vol. 10, no. 4, Article ID e0122854, 2015.

[4] A. A. Ball, "CONSURF. Part one: introduction of the conic lofting tile," Computer-Aided Design, vol. 6, no. 4, pp. 243-249, 1974.
[5] S. A. A. Karim, "Data interpolation using rational cubic Ball spline with three parameters," in Proceedings of the 4th International Conference on Fundamental and Applied Sciences (ICFAS '16), August 2016.

[6] S. A. A. Karim, "Positivity preserving interpolation by using rational cubic ball spline," Jurnal Teknologi, vol. 78, no. 11, pp. 141-148, 2016.

[7] K. W. Brodlie and S. Butt, "Preserving convexity using piecewise cubic interpolation," Computers and Graphics, vol. 15, no. 1, pp. $15-23,1991$.

[8] M. Z. Hussain, M. Sarfraz, and T. S. Shaikh, "Shape preserving rational cubic spline for positive and convex data," Egyptian Informatics Journal, vol. 12, no. 3, pp. 231-236, 2011.

[9] M. Z. Hussain, M. Hussain, and A. Waseem, "Shape-preserving trigonometric functions," Computational \& Applied Mathematics, vol. 33, no. 2, pp. 411-431, 2014.

[10] H. Akima, "A new method of interpolation and smoth curve fitting based on local procedures," Journal of the ACM, vol. 17, no. 4, pp. 598-602, 1970.

[11] J. W. Schmidt and W. Hess, "Positivity of cubic polynomials on intervals and positive spline interpolation," BIT Numerical Mathematics, vol. 28, no. 2, pp. 340-352, 1988.

[12] S. A. Karim, V. P. Kong, and A. Saaban, "Positivity preserving interpolation using rational bicubic spline," Journal of Applied Mathematics, vol. 2015, Article ID 572768, 15 pages, 2015.

[13] D. Han, "Comparison of commonly used image interpolation methods," in Proceedings of the 2nd International Conference on Computer Science and Electronics Engineering (ICCSEE '13), pp. 1556-1559, March 2013.

[14] M. Z. Hussain, S. Abbas, and M. Irshad, "Quadratic trigonometric B-spline for image interpolation using GA," PLoS ONE, vol. 12, no. 6, p. e0179721, 2017.

[15] S. A. Karim and V. P. Kong, "Shape preserving interpolation using $\mathrm{C}^{2}$ rational cubic spline," Journal of Applied Mathematics, Article ID 4875358, 14 pages, 2016. 


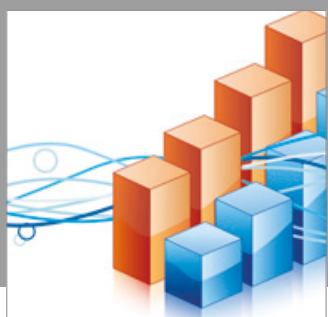

Advances in

Operations Research

vatersals

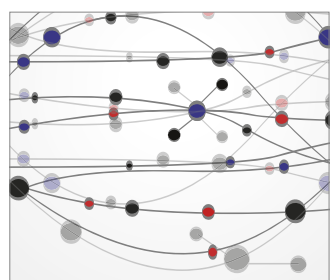

\section{The Scientific} World Journal
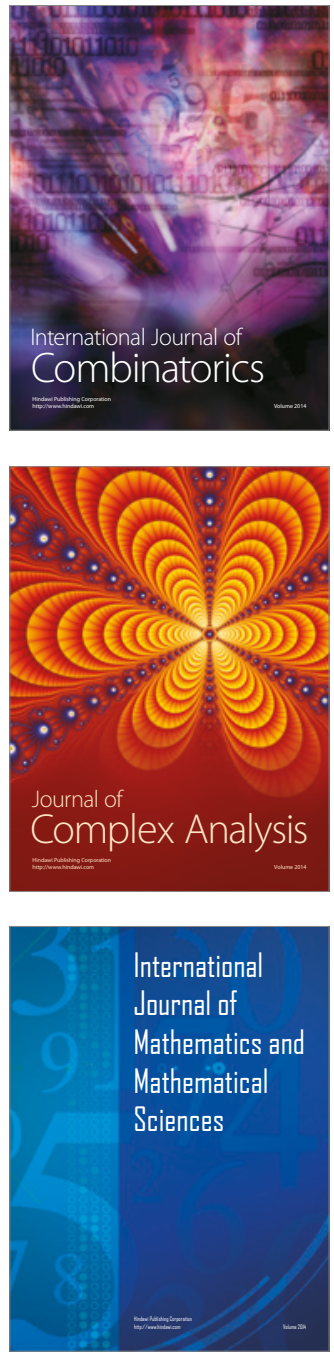
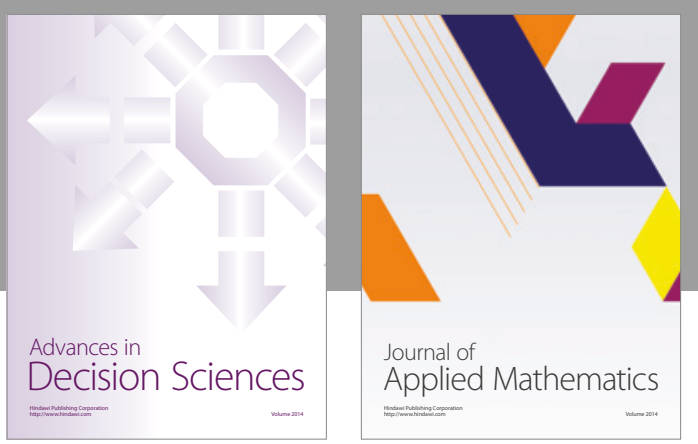

Algebra

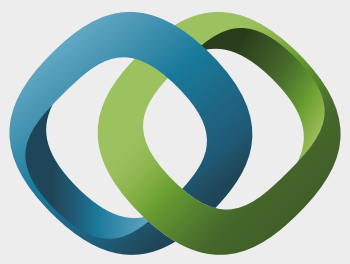

\section{Hindawi}

Submit your manuscripts at

https://www.hindawi.com
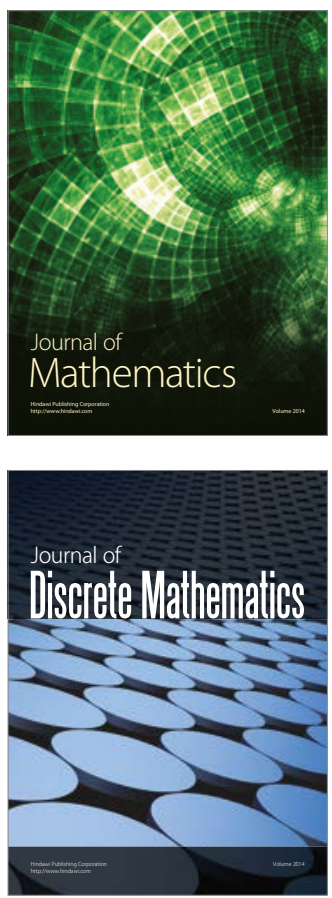

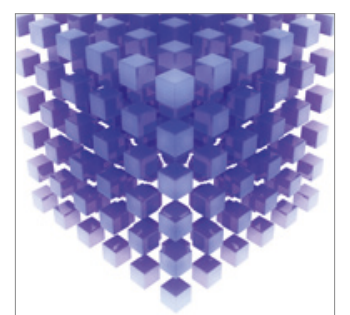

Mathematical Problems in Engineering
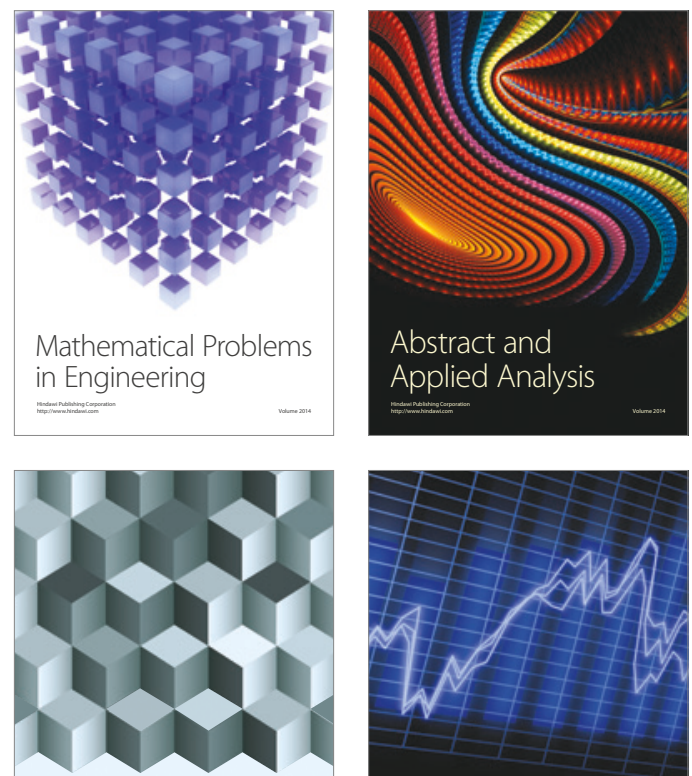

Journal of

Function Spaces

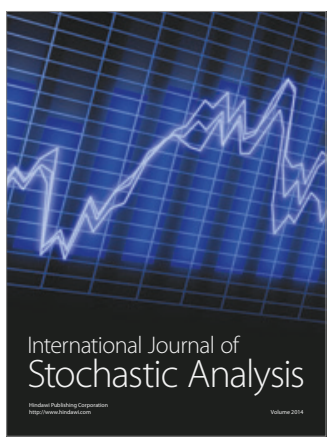

Probability and Statistics
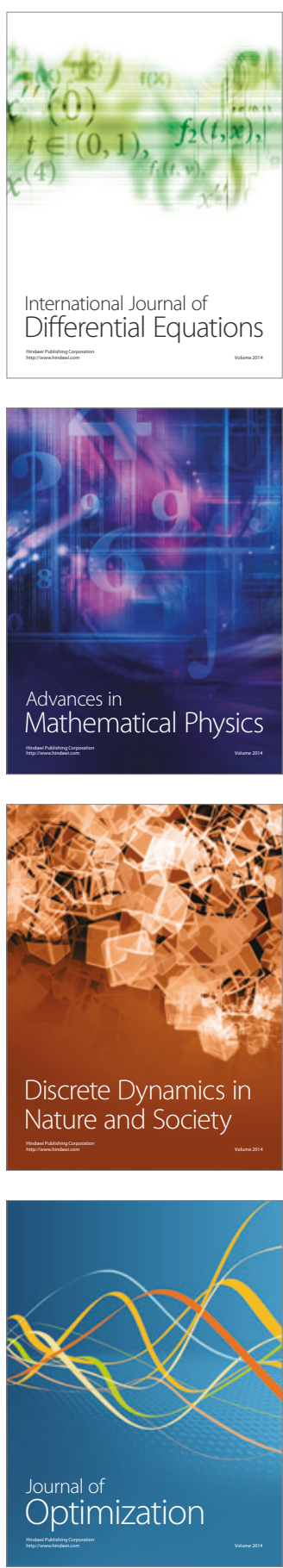